\title{
Polaprezinc protects normal intestinal epithelium against exposure to ionizing radiation in mice
}

\author{
SOICHI ODAWARA ${ }^{1}$, HIROSHI DOI ${ }^{1}$, TOSHIYUKI SHIKATA ${ }^{2}$, KAZUHIRO KITAJIMA ${ }^{1}$, \\ HITOMI SUZUKI ${ }^{1}$, YASUE NIWA ${ }^{3}$, KENGO KOSAKA $^{1}$, KAZUO TARUTANI ${ }^{1}$, \\ TOHRU TSUJIMURA ${ }^{4}$, NORIHIKO KAMIKONYA ${ }^{1}$ and SHOZO HIROTA $^{1}$
}

${ }^{1}$ Department of Radiology, Hyogo College of Medicine, Nishinomiya, Hyogo 663-8501; ${ }^{2}$ Department of Pharmacy, Hyogo College of Medicine Sasayama Medical Center, Sasayama, Hyogo 669-2321; ${ }^{3}$ Department of

Therapeutic Radiology, Uji-Tokushukai Medical Center, Uji, Kyoto 611-0041; ${ }^{4}$ Department of Pathology, Hyogo College of Medicine, Nishinomiya, Hyogo 663-8501, Japan

Received February 1, 2016; Accepted June 9, 2016

DOI: $10.3892 / \mathrm{mco} .2016 .983$

\begin{abstract}
Polaprezinc (PZ), an antiulcer drug, has been reported to have antioxidant effects. The purpose of the present study was to assess the radioprotective effects of $\mathrm{PZ}$ in the normal intestine of C57BL/6J mice. PZ was orally administered at $100 \mathrm{mg} / \mathrm{kg}$ body weight in the drinking water. Firstly, the present study compared the survival of normal intestinal crypt epithelial cells with mice that received PZ prior to or following irradiation. Next, the present study examined the sequential changes of the incidence of apoptosis in the normal intestine of mice that received irradiation. The mice that received PZ prior to irradiation demonstrated a stronger protective effect on the normal intestine compared with those that received PZ after irradiation. The present study therefore administrated PZ $2 \mathrm{~h}$ before irradiation in the subsequent experiments. The mice receiving PZ developed fewer apoptotic cells in the duodenum, jejunum and ileum. Radiation-induced cell death occurred with a peak at position 10 or lower from the base of the crypt axis, and was subsequently reduced by PZ treatment. Pretreatment with PZ protected the normal intestinal tissues from radiation-induced apoptosis.
\end{abstract}

\section{Introduction}

Radiotherapy serves an important role in cancer treatment. Radiation-induced gastrointestinal disorder is the most common complication of toxicity due to the treatment for malignancies in the abdomen and pelvis, which often reduces the quality of life (QOL) for patients and occasionally limits the therapeutic dose that can be used for treating abdominal

Correspondence to: Dr Hiroshi Doi, Department of Radiology, Hyogo College of Medicine, 1-1 Mukogawa-cho, Nishinomiya, Hyogo 663-8501, Japan

E-mail: h-doi@hyo-med.ac.jp

Key words: polaprezinc, radioprotectant, radiotherapy, colitis and pelvic tumors with radiotherapy. Modern radiotherapeutic modalities, including intensity-modulated radiation therapy and proton therapy, can significantly decrease the toxicities of radiotherapy and can benefit the outcomes (1). Amifostine has been recommended for the prevention of severe radiation-induced toxicities and is the only radioprotectant that has been approved by the US Food and Drug Administration (2). However, the daily use of amifostine has been limited due to its hematological and gastrointestinal toxicity.

Polaprezinc (PZ) is an antiulcer drug and a chelating compound consisting of a zinc ion, L-carnosine, a $\beta$-alanine dipeptide and L-histidine. It was previously reported that PZ exerts antioxidant effects and scavenges free radicals (3-5). Our previous study reported the efficacy of $\mathrm{PZ}$ for acute radiation proctitis in an animal model and demonstrated that PZ has an anti-inflammatory effect following exposure to radiation (6). However, the radioprotective effects of $\mathrm{PZ}$ have been reported in only a limited number of reports and remain unclear (6-9).

The purpose of the present study was to examine the optimal timing to administer PZ and the pattern of apoptosis in the normal intestine. It is important that premedication of the clinical medicine enables the prevention of radiation injuries.

\section{Materials and methods}

Animal models and reagents. Male C57BL/6J mice (8-weeks-old; weight, $\sim 20 \mathrm{~g}$ ) were used in the present study. The mice were purchased from Charles River Laboratories Japan, Inc. (Kanagawa, Japan) and were acclimated for 7 days. They were housed 4-5/cage and fed a laboratory rodent pellet formula and tap water ad libitum. Mice were maintained at a constant temperature of $22^{\circ} \mathrm{C} \pm 0.5^{\circ} \mathrm{C}$, a humidity of $50 \% \pm 5 \%$ and were exposed to $12 \mathrm{~h}$ light/dark cycles. The Hyogo College of Medicine Institutional Animal Care and Use Committee approved all animal procedures prior to the initiation of the project (nos. 13-047 and 13-070). The mice orally received $100 \mathrm{mg} / \mathrm{kg}$ body weight $\mathrm{PZ}$ in the drinking water. The mice were irradiated at a dose rate of $200 \mathrm{cGy} / \mathrm{min}$ using a 150 kVp X-ray unit (Hitachi MBR-1520; Hitachi, Tokyo, 
Japan; $20 \mathrm{~mA} ; 150 \mathrm{kV}$ ). For dosimetry, a probe connected to an electrometer system was placed close to the target site.

Radioprotection of $P Z$ on normal intestine. Prior to the experiments, the present study confirmed that the agents were retained in the whole intestine using an imaging examination with sodium amidotrizoate $(100 \mathrm{mg} / \mathrm{ml}) 2 \mathrm{~h}$ after the oral administration of PZ (Zeria Pharmaceutical Co., Ltd., Tokyo, Japan; data not shown). To examine the optimal timing of the administration of PZ for the protective effect on the normal intestine, the present study used an established animal model that measured the intestinal stem cell survival (as the number of crypts/cross section) after ionizing radiation (IR).

The detailed procedure for the intestinal crypt cell survival analysis following irradiation has been described previously (10). Briefly, the mice were divided into the following three groups: Group A, mice that received IR without PZ; group $\mathrm{B}$, mice that received PZ $2 \mathrm{~h}$ before IR; group C, mice that received PZ $2 \mathrm{~h}$ after IR (n=3/group). The mice were irradiated with a total body irradiation (TBI) of $15 \mathrm{~Gy}$ in a single fraction. Three days after radiation, the mice were sacrificed by injection of pentobarbital $(200 \mathrm{mg} / \mathrm{kg})$ in the abdominal cavity, and the duodenum, jejunum and ileum were removed, fixed and stained with hematoxylin and eosin (H\&E). Surviving crypts with $\geq 10$ cells for each cross-section were counted using microscopy.

In the subsequent studies, based on the results, the mice were divided into two groups: Mice that received oral administration of PZ $2 \mathrm{~h}$ prior to IR [PZ (+) group] and mice that were treated with drinking water without PZ [PZ (-) group]. The samples were harvested for a pathological evaluation and were immediately fixed in $10 \%$ neutral buffered formalin solution. The intestine was divided into the duodenum, jejunum and ileum, and was subsequently submitted for a histological analysis. All slides were stained with H\&E and examined using light microscopy. The radioprotective effects of PZ in these two murine groups were evaluated.

To examine the suppressive effect of $\mathrm{PZ}$ on the apoptosis caused by IR in the normal intestine, the mice received TBI of $2 \mathrm{~Gy}$ in a single fraction and were subsequently sacrificed at 0,4 and $8 \mathrm{~h}$ following IR. The group for $0 \mathrm{~h}$ used two mice for the PZ (-) group and two for the PZ (+) group, and the group for 4 and $8 \mathrm{~h}$ used four mice for the PZ (-) group and four for the PZ (+) group. The duodenum, jejunum, ileum and rectum were harvested for pathological examination. The present study defined apoptotic cells as epithelial cells with apoptotic fragments in the H\&E stained tissue sections. In addition, the proportion of apoptotic cells in each crypt was analyzed to determine an apoptotic index. The position of apoptotic cells were determined by the numbering from the bottom of the crypt cells.

Statistical analysis. The data are expressed as the means, with the range in parentheses, unless otherwise indicated. A statistical analysis was performed to compare the differences between the two or three groups. For the two groups, the parametric data were analyzed using an unpaired two-tailed $\mathrm{F}$ test and the two-tailed Student's t-test. For the three groups, the parametric data were analyzed using the Bartlett test and by a one-way analysis of variance.

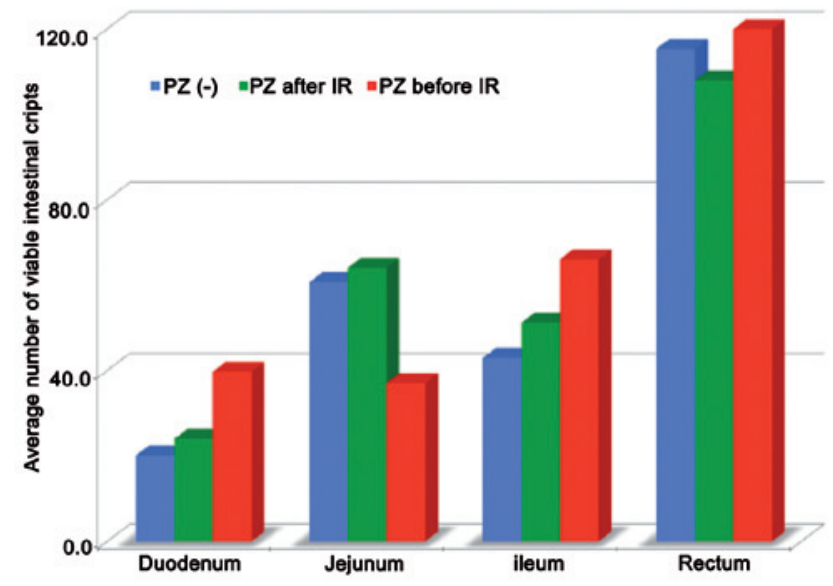

Figure 1. Optimal timing of the administration of PZ prior to or following 15 Gy total body IR. Treatment with PZ increased the number of viable intestinal crypts in the normal intestine and the oral administration of PZ prior to IR revealed marked radioprotective effects on the intestinal crypt cells compared with those observed following IR. PZ, polaprezinc; IR, ionizing radiation.

When significant differences in the three groups were observed, a multiple comparison using the Tukey-Kramer method was performed. In addition, a risk of 5\% was defined as a significant difference between the two groups. The StatMate IV software program (ATMS Co., Ltd., Tokyo, Japan) was used to perform statistical analysis. $\mathrm{P}<0.05$ was considered to indicate a statistically significant difference.

\section{Results}

The present study performed experiments to study the effect of $\mathrm{PZ}$ on the intestinal crypt survival following 15 Gy TBI. The results demonstrated that the administration of PZ decreased the radiation-induced cell death of crypt stem cells in the duodenum, jejunum and ileum. In addition, PZ treatment protected the crypt cells against TBI and increased the number of viable crypt cells in the mice that received PZ prior to IR compared with those that received PZ after IR (Fig. 1).

The average number of viable intestinal crypts in groups $\mathrm{A}$, $\mathrm{B}$ and $\mathrm{C}$ was 20.2, 24.1 and 39.8 in the duodenum $(\mathrm{P}=0.04)$, and $43,51.3$ and 66.1 in the ileum $(\mathrm{P}=0.02)$, respectively (Fig. 1).

In addition, according to the multiple comparison test method, significant differences existed between groups A and $\mathrm{C}$ in both the duodenum and ileum. Mice that received premedication of PZ exhibited significantly more viable intestinal stem cells in the crypts, compared with those administered PZ after IR (Fig. 1).

$\mathrm{PZ}$ was administered $2 \mathrm{~h}$ prior to IR, according to the above mentioned data. In the pathological examination, apoptotic cells were observed (Fig. 2). The number of apoptotic cells in the small intestine peaked at $8 \mathrm{~h}$ following IR and the oral administration of $\mathrm{PZ}$ protected the normal intestinal cells from apoptosis caused by IR (Fig. 3). The apoptotic index in the PZ (-) group was 0.056, 0.106 and 0.084 in the duodenum, jejunum and ileum, respectively. The apoptotic index in the PZ (+) group was $0.022,0.044$ and 0.052 in the duodenum, jejunum and ileum, respectively. PZ tended to reduce the apoptosis in the duodenum and jejunum (Fig. 3), however not 


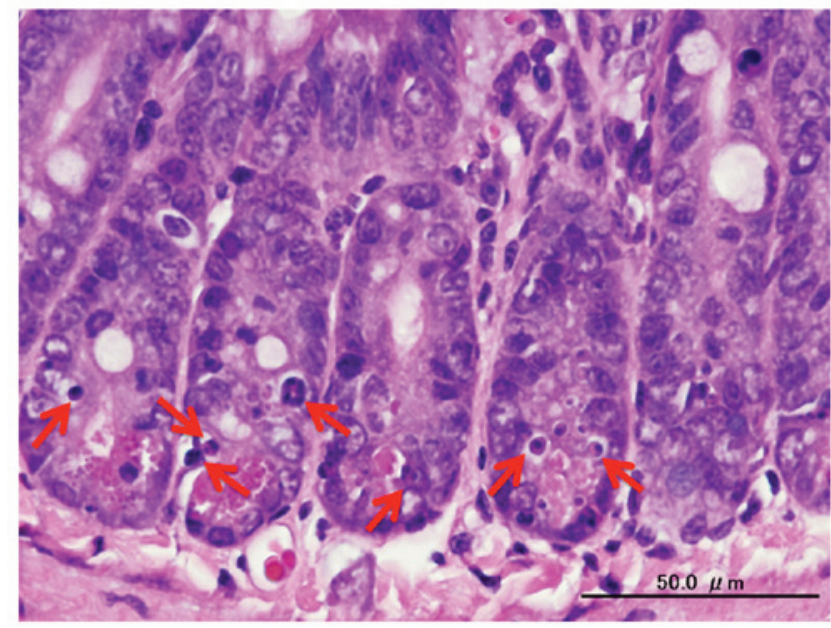

Figure 2. Hematoxylin and eosin staining of cells $4 \mathrm{~h}$ after irradiation. At $4 \mathrm{~h}$ after 15 Gy total body irradiation, intestinal crypt cells that contained apoptotic (micronuclei) bodies in the pathological findings using hematoxylin and eosin staining were defined as apoptotic cells (arrows; scale bar, $50 \mu \mathrm{m}$ ).

the ilium. The P-values between the PZ (+) and PZ (-) groups were $0.07,0.01$ and 0.12 in the duodenum, jejunum, and ileum, respectively.

The positions of high apoptotic frequency were observed in lower positions of each crypt in the intestine. The mice that received $\mathrm{PZ}$ exhibited less apoptotic cells in each position of crypts (Figs. 4 and 5).

\section{Discussion}

Radiotherapy is a significant treatment option for malignancy and can improve patient survival. Gastrointestinal toxicity commonly occurs as a complication of radiotherapy for malignancy in the abdomen and pelvis, which significantly decreases a patient's QOL $(2,11)$. In addition, no standardized treatment options are currently available to prevent gastrointestinal toxicity (2).

PZ, which is a chelating compound consisting of a zinc ion, L-carnosine and L-histidine, has been reported to possess superoxide scavenging activity (3). It has also been previously reported that $\mathrm{PZ}$ exerts its effects via a variety of cytoprotective mechanisms, including the suppression of lipid peroxidation, reduction of the levels of various cytokines, inhibition of superoxide generation and promotion of the restoration of the gastric mucosa (3-5). Therefore, the efficacy of $\mathrm{PZ}$ for radiation-induced mucositis appears promising. Our previous study reported the efficacy of PZ for acute radiation proctitis in a rat model and demonstrated the anti-inflammatory effect of $\mathrm{PZ}$ on radiation-induced mucosal damage (6). In addition, the clinical use of PZ has been reported to be feasible and can increase a patient's QOL during radiotherapy $(8,9)$.

In the present study, the pretreatment of PZ revealed improved protective effects in the mice that received $\mathrm{PZ}$ prior to radiation exposure compared with those that received $\mathrm{PZ}$ after radiation exposure and those that were irradiated without PZ. The present study therefore administered PZ $2 \mathrm{~h}$ prior to IR. This is the first report, to the best of our knowledge, that
A

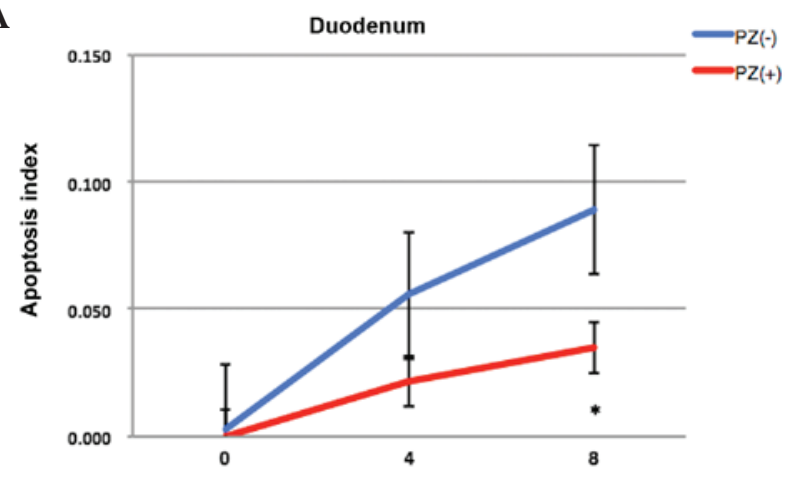

B

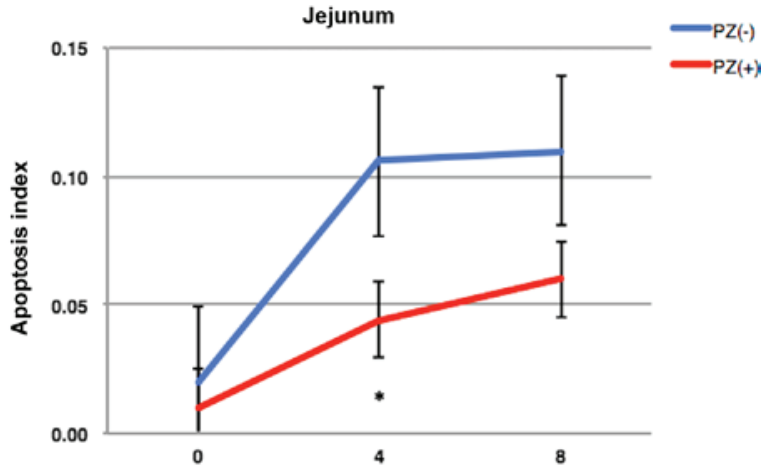

C

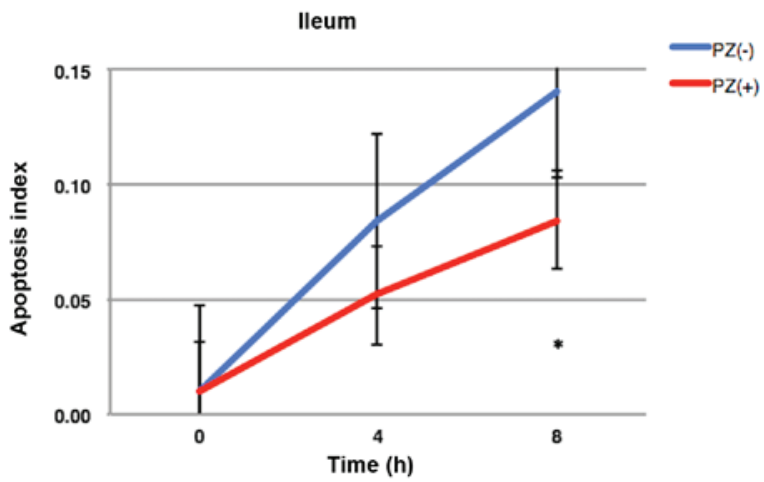

Figure 3. Sequential changes of apoptotic indexes in the (A) duodenum, (B) jejunum, and (C) ileum at the various times (0, 4 and 8 h) following 2 Gy irradiation. $\mathrm{PZ}$ reduced apoptosis in all intestinal sites and time points. PZ, polaprezinc.

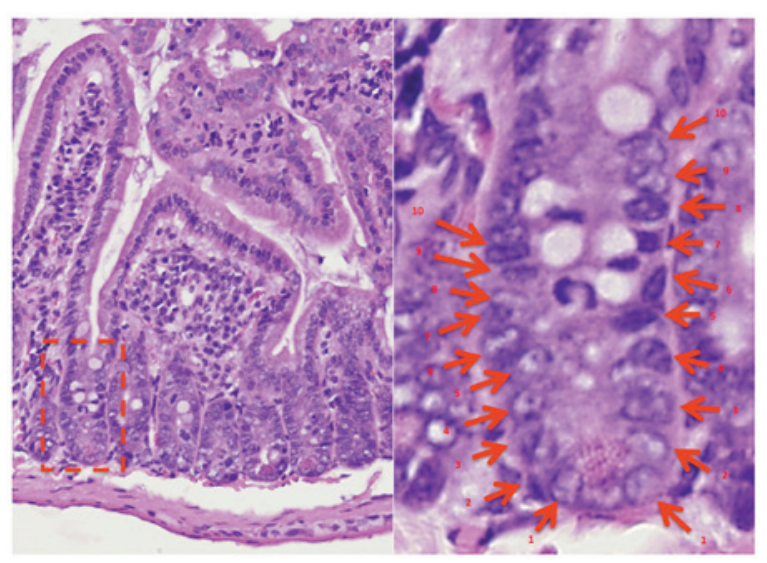

Figure 4. Apoptotic cell positions were highlighted on the hematoxylin and eosin stained tissues (magnification, $\mathrm{x} 20$ ). The position of apoptosis was determined, as shown in the figure from the bottom of the crypt. 
A

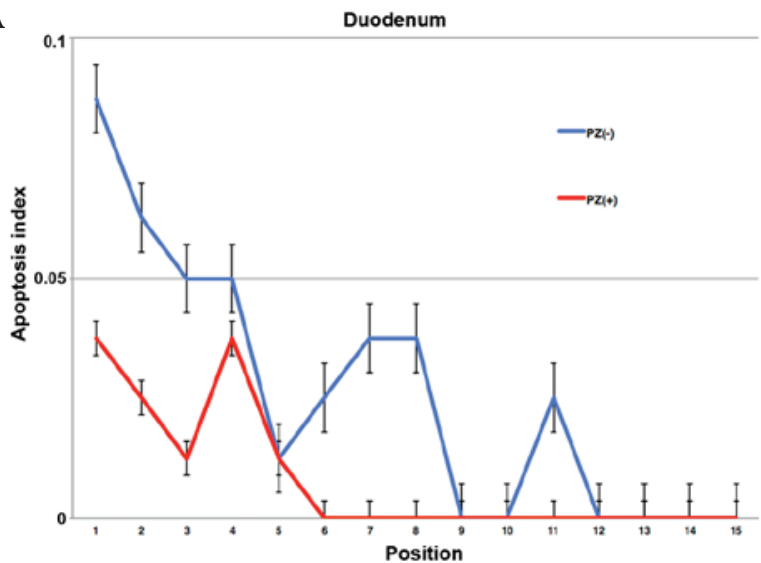

B

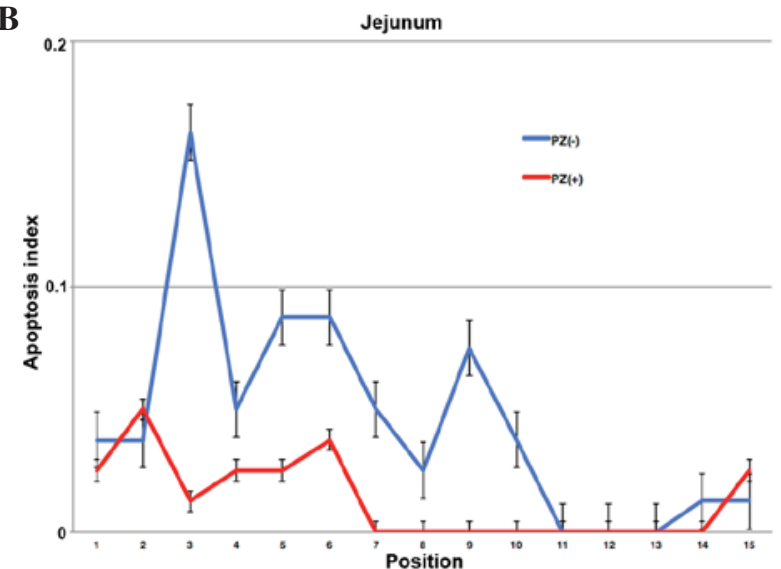

C

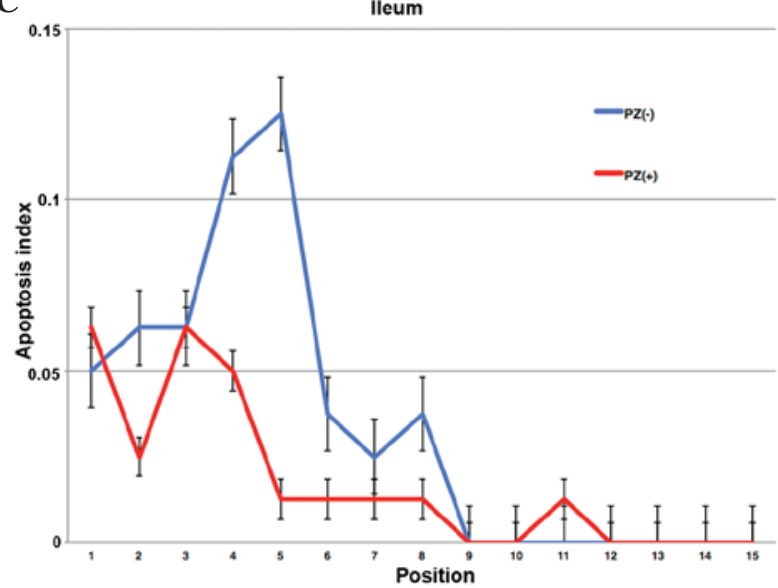

Figure 5. Apoptotic index of cells in each position in the crypt. An increased incidence of apoptosis was observed at positions 1-10 from the base of the crypt axis in the (A) duodenum, (B) jejunum and (C) ileum at $4 \mathrm{~h}$ following irradiation. PZ, polaprezinc.

demonstrated the optimal timing for PZ treatment as a radioprotectant. Radiotherapy is generally performed in a fractionated schedule with the aim of maximizing the anti-tumor effect and minimizing the toxicity of normal organs in the clinical practice. However, the present study used a single fraction schedule to examine the efficacy of PZ. Therefore, the optimization of the administration of PZ and IR must be evaluated in future studies.

Matsuu-Matsuyama et al (7) have reported that PZ can protect the normal intestine from radiation-induced apoptosis via the suppression of $\mathrm{p} 53, \mathrm{p} 21$ and B-cell associated $\mathrm{X}$ protein genes. However, the mechanism of the radioprotective effect of PZ treatment for radiation-induced mucosal damages remains to be investigated in greater detail. The present study examined the potential differences in the sensitivity of PZ treatment in various positions of the crypts in the intestine since intestinal stem cells, which are critical drivers of epithelial homeostasis and regeneration, have been reported to localize to the lower areas in each crypt (12). It was revealed that radiation induced apoptosis in the low positions from the base of the crypt axis, which was reduced following PZ treatment. In addition, the present study also demonstrated the radioprotective effect of PZ in the established-intestinal crypt stem cell assay. According to these results, PZ protects the intestinal stem cells from cell death caused by IR and can reduce gastrointestinal toxicity associated with radiotherapy.

Cytoprotection of radioprotectants can include joint binding between the tumor cells and normal cells, since reactive oxygen species are believed to serve a significant role in radiotherapy delivered by photons. There is, however, little data of the negative effects of $\mathrm{PZ}$ use for tumor control and the survival outcomes of the patients who received PZ as a supportive treatment have been poorly understood. However, no direct evidence has suggested that the use of PZ can lead to inferior tumor outcomes in patients with malignancies $(8,9)$. Watanabe et al $(8)$ reported that the initial responses of tumors to radiotherapy were not affected by the PZ intake in a randomized clinical trial. In addition, our previous study recently reported that patients who received a PZ-containing rinse showed no inferior survival outcomes compared with those that did not received PZ in a retrospective study (9). However, further investigations are required prior to a large clinical trial.

The present study administered PZ $2 \mathrm{~h}$ prior to IR in order to distribute PZ to whole intestine, according to the findings from our preliminary data. In addition, Myagmarjalbuu et al (13) have reported the gastrointestinal transit time was $2 \mathrm{~h}$ in mice. It has been previously reported that PZ causes its pharmacological effect by adhesion to damaged mucosa $(4,6)$. However, the time between the administration of PZ and IR can increase the serum zinc concentration (12). Therefore, further studies to determine the mechanism of the radioprotective effects of $\mathrm{PZ}$ are required.

In conclusion, premedication with $\mathrm{PZ}$ protected the normal intestinal tissues from IR-induced apoptosis.

\section{Acknowledgements}

The authors would like to thank Ms. Michiko Kakihana (Department of Pathology, Hyogo College of Medicine, Nishinomiya, Hyogo, Japan) for her valuable technical assistance and Mr. Daisuke Nagata (Institute of Experimental Animal Sciences, Hyogo College of Medicine, Nishinomiya, Hyogo, Japan). The present study was supported, in part, by funding from the Grants-in-Aid for Scientific Research (no. 24591854) and unrestricted funding from Zeria Pharmaceutical Co., Ltd.

\section{References}

1. Zelefsky MJ, Levin EJ, Hunt M, Yamada Y, Shippy AM, Jackson A and Amols HI: Incidence of late rectal and urinary toxicities after three-dimensional conformal radiotherapy and intensity-modulated radiotherapy for localized prostate cancer. Int J Radiat Oncol Biol Phys 70: 1124-1129, 2008. 
2. Lalla RV, Bowen J, Barasch A, Elting L, Epstein J, Keefe DM, McGuire DB, Migliorati C, Nicolatou-Galitis O, Peterson DE, et al: MASCC/ISOO clinical practice guidelines for the management of mucositis secondary to cancer therapy. Cancer 120: 1453-1461, 2014

3. Yoshikawa T, Naito Y, Tanigawa T, Yoneta T and Kondo M The antioxidant properties of a novel zinc-carnosine chelate compound, N-(3-aminopropionyl)-L-histidinato zinc. Biochim Biophys Acta 1115: 15-22, 1991.

4. Yoshikawa T, Yamaguchi T, Yoshida N, Yamamoto H, Kitazumi S Takahashi S, Naito Y and Kondo M: Effect of Z-103 on TNB-induced colitis in rats. Digestion 58: 464-468, 1997.

5. Ohkawara T, Nishihira J, Nagashima R, Takeda H and Asaka M: Polaprezinc protects human colon cells from oxidative injury induced by hydrogen peroxide: Relevant to cytoprotective heat shock proteins. World J Gastroenterol 12: 6178-6181, 2006.

6. Doi H, Kamikonya N, Takada Y, Fujiwara M, Tsuboi K, Inoue H, Tanooka M, Nakamura T, Shikata T, Tsujimura T and Hirota S: Efficacy of polaprezinc for acute radiation proctitis in a rat model. Int J Radiat Oncol Biol Phys 80: 877-884, 2011.

7. Matsuu-Matsuyama M, Shichijo K, Okaichi K, Nakayama T, Nakashima M, Uemura T, Niino D and Sekine I: Protection by polaprezinc against radiation-induced apoptosis in rat jejunal crypt cells. J Radiat Res 49: 341-347, 2008
8. Watanabe T, Ishihara M, Matsuura K, Mizuta K and Itoh Y: Polaprezinc prevents oral mucositis associated with radiochemotherapy in patients with head and neck cancer. Int J Cancer 127: 1984-1990, 2010

9. Doi H, Fujiwara M, Suzuki H, Niwa Y, Nakayama M, Shikata T, Odawara S, Takada Y, Kimura T, Kamikonya N and Hirota S: Polaprezinc reduces the severity of radiation-induced mucositis in head and neck cancer patients. Mol Clin Oncol 3: 381-386, 2015.

10. Withers HR and Elkind MM: Radiosensitivity and fractionation response of crypt cells of mouse jejunum. Radiat Res 38: 598-613, 1969.

11. Kavanagh BD, Pan CC, Dawson LA, Das SK, Li XA, Ten Haken RK and Miften M: Radiation dose-volume effects in the stomach and small bowel. Int J Radiat Oncol Biol Phys 76 (Suppl 3): S101-S107, 2010.

12. Barker N: Adult intestinal stem cells: Critical drivers of epithelial homeostasis and regeneration. Nat Rev Mol Cell Biol 15: 19-33, 2014.

13. Myagmarjalbuu B, Moon MJ, Heo SH, Jeong SI, Park JS, Jun JY, Jeong YY and Kang HK: Establishment of a protocol for determining gastrointestinal transit time in mice using barium and radiopaque markers. Korean J Radiol 14: 45-50, 2013. 